IFT-UAM/CSIC-01-02

hep-th/0101181

\title{
Non-critical Non-singular Bosonic Strings, Linear Dilatons and Holography
}

\author{
Enrique Álvarez, César Gómez ^, Lorenzo Hernández, and Pedro Resco \\ Instituto de Física Teórica, C-XVI, Universidad Autónoma de Madrid \\ E-28049-Madrid, Spain \\ and \\ Departamento de Física Teórica, C-XI, Universidad Autónoma de Madrid \\ E-28049-Madrid, Spain \\ - I.M.A.F.F., C.S.I.C., Calle de Serrano 113 \\ E-28006-Madrid, Spain
}

\begin{abstract}
$A d S_{5}$ with linear dilaton and non vanishing $B$-field is shown to be a solution of the non critical string beta function equations. A non critical $(D=5)$ solution interpolating between flat space-time and $A d S_{5}$, with asymptotic linear dilaton and non vanishing $B$ field is also presented. This solution is free of space-time singularities and has got the string coupling constant everywhere bounded. Both solutions admit holographic interpretation in terms of $\mathcal{N}=0$ field theories. Closed string tachyon stability is also discussed.
\end{abstract}

\footnotetext{
${ }^{1}$ Unidad de Investigación Asociada al Centro de Física Miguel Catalán (C.S.I.C.)
} 


\section{Introduction}

Much effort has recently been devoted to research in Anti de Sitter (AdS) string backgrounds. The reason is at least, twofold. First of all, holography (cf. [7], [6] [10]) relates bulk gravitational physics in $A d S_{5}$ with some conformal field theory (CFT) at the boundary. In addition, AdS is by far the simplest geometry where the ideas of Randall-Sundrum [9] on four-dimensional confinement of gravity and hierarchy generation can be implemented.

The ten-dimensional background $A d S_{5} \times S_{5}$ is a well-known background geometry for the type $I I B$ string description of the near horizon limit of D3 branes. In order for it to become a consistent string background, a self-dual Ramond-Ramond (RR) five-form has to be turned on. The five sphere $S_{5}$ plays the rôle of an internal manifold, and is associated with the extra scalars required by $\mathcal{N}=4$ supersymmetry.

One of the main motivations of the present paper stems from the natural question as to whether $A d S_{5}$ can define a non-critical string background. We will actually show this to be the case with euclidean signature, provided both an appropiate dilaton and Kalb-Ramond field are turned on.

A simple extrapolation of the holographic principle to the present situation suggests that this background should be dual with some four-dimensional, non-supersymmetric, $\mathcal{N}=0$, CFT on the boundary.

Actually, the simplest non-critical bosonic string background is the linear dilaton [8], with a dilaton field:

$$
\Phi= \pm Q r
$$

living in flat $d$-dimensional Minkowski (or euclidean) spacetime:

$$
d s^{2}=d \vec{x}_{(1, d-1)}^{2}+d r^{2}
$$

with

$$
q \equiv \frac{d-25}{6 \alpha^{\prime}} \equiv-Q^{2}
$$


(where $Q \in \mathbb{R}$ whenever $D \equiv d+1<26$ ).

It is plain that we actually have two $\mathbb{Z}_{2}$-related different potential vacua corresponding to the two admissible signs in (11). Bearing in mind a holographic interpretation of the coordinate $r$, it is natural to ask whether there are topologically non-trivial configurations which interpolate between these two vacua, i.e., with a dilaton behavior of the type

$$
\begin{aligned}
& \Phi(r)_{r \rightarrow \infty} \sim-Q r \\
& \Phi(r)_{r \rightarrow-\infty} \sim+Q r
\end{aligned}
$$

The answer is in the affirmative (again, only with euclidean signature). The first interpretation of this solution that comes to mind is that of a sort of tunneling between the two $\mathbb{Z}_{2}$-related linear dilaton behaviors defined above.

Asymptotically $(r \rightarrow \infty)$ this interpolating solution is just the linear dilaton in flat space-time with weak string coupling constant. At $r \rightarrow-\infty$ the solution becomes $A d S_{5}$ with linear dilaton, but again with weak string coupling constant ( see Fig 2 ). In addition the solution is free of singularities with a bounded curvature everywhere.

It is of course interesting to look for the holographic interpretation of this solution.

String backgrounds that behave asymptotically at $r \rightarrow \infty$ as

$$
\begin{aligned}
& d s^{2}=d \vec{x}_{(1, d-1)}^{2}+d r^{2}+d s^{2}(M) \\
& g_{s}^{2}=e^{-\Delta r}
\end{aligned}
$$

where $\Delta$ is a constant and $M$ a compact internal manifold, trivially fibered on the flat $d+1$ dimensional space-time, have been first considered, from the holographic point of view, in reference [1].

It was there argued that this type of string backgrounds are holographic. This fact can be easily understood by considering the metric in the Einstein frame. In this frame ,and thanks to the linear dilaton, the $d+1$ dimensional space-time metric becomes effectively $A d S$ metric, which in particular implies the necessary condition of holography, namely 
that timelike geodesics never reach the boundary. According to [1] the holographic dual of these backgrounds is a non local little string theory. One of the main problems of linear dilaton backgrounds of type (5) considered in [1] is how to regulate the divergences in the strong coupling region $(r \rightarrow-\infty)$.

At this point it is worth noticing that the interpolating solution we present in this paper behaves asymptotically exactly as above (5), up to the fact that we have not any internal compact manifold]. Moreover our solution automatically regulates the divergence at $r \rightarrow-\infty$ by becoming, in a smooth way, $A d S_{5}$ with weak string coupling constant. From the holographic point of view the boundary theory could be a non local little string theory with $\mathcal{N}=0$ supersymmetry. If this is the case it would be very interesting to understand if this hypothetical non locality alluded to above could be due in our case to the non vanishing expectation value for the Kalb-Ramond field.

The other solution we find, namely $A d S_{5}$ with linear dilaton and B-field is also interesting by itself, from the point of view of holography. The most natural candidate for the boundary theory would be of course a $\mathcal{N}=0$ theory coupled to non vanishing B-field. In the context of $\mathcal{N}=8$ gauged supergravity there are two non trivial $A d S_{5}$ minima associated with expectation values for the $\underline{10}$ and the $\underline{20}$ multiplets [5]. Holographically these two vacua should correspond to $\mathcal{N}=0$ four dimensional conformal field theories. In these cases the string background is critical $A d S_{5} \times W_{5}$ but with the internal manifold $W$ fibered in a non trivial way on the holographic coordinate. It would be interesting to study if there exist any relation between these $\mathcal{N}=0$ backgrounds predicted by $\mathcal{N}=8$ supergravity and the $A d S_{5}$ solution with non vanishing $\mathrm{B}$ described in this paper.

Finally let us just mention that the interpolating solution we present is only valid with euclidean signature. This is the reason we tend to interpret it as a sort of tunneling effect.

\footnotetext{
${ }^{2}$ Although we could, just by introducing toroidal spectator dimensions
} 


\section{$2 A d S_{5}$ and the Bosonic String}

The first step in determining whether strings can live in a given background is to check the vanishing of the Weyl anomaly coefficients, that is:

$$
\begin{aligned}
& R_{A B}+2 \nabla_{A} \nabla_{B} \Phi-\frac{1}{4} H_{A C D} H_{B}^{C D}=0 \\
& -\frac{1}{2} \nabla^{C} H_{C A B}+\nabla^{C} \Phi H_{C A B}=0 \\
& q+(\nabla \Phi)^{2}-\frac{1}{2} \nabla^{2} \Phi-\frac{1}{24} H^{2}=0
\end{aligned}
$$

We have denoted by capital latin letters spacetime indices $(A, B, \ldots=0,1 \ldots D-1 \equiv d)$, and by greek letters Poincaré indices $(\mu, \nu, \ldots=0, \ldots, d-1)$.

We intend to look for backgrounds in which the metric part enjoys Poincaré invariance, that is

$$
d s^{2}=a(r) d \vec{x}_{(1, d-1)}^{2}+d r^{2}
$$

But allowing for nontrivial dilaton and Kalb-Ramond background. The dilaton background still preserves $I O(1, d-1)$ invariance because it depends on the holographic coordinate only, $\Phi=\Phi(r)$. But any nontrivial antisymmetric background necessarily breaks it (in the sense that $£(k) H_{A B C} \neq 0$ for all non translational Killing vectors $k$ ); the only remaining unbroken symmetry being the four dimensional translation group, $\mathbb{T}^{4}$.

Let us begin by assuming an AdS ansatz,

$$
a(r)=e^{2 Q r}
$$

dressing it with a linear dilaton,

$$
\Phi(r)=Q r+\Phi_{0}
$$

and allowing for a nontrivial Kalb-Ramond field,

$$
b_{\mu \nu}(r)=c_{\mu \nu} e^{2 \Phi_{0}} a(r)+b_{\mu \nu}^{0}
$$


where both $c_{\mu \nu}$ and $b_{\mu \nu}^{0}$ are constant tensors.

Remarkably enough, this ansatz can easily be shown to be a solution, provided only that the constants obey the following relationships:

$$
\begin{aligned}
& \eta^{\alpha \beta} c_{\mu \alpha} c_{\nu \beta}=0(\mu \neq \nu) \\
& (2-d) \eta_{\mu \nu}=2 e^{4 \Phi_{0}} \eta^{\alpha \beta} c_{\mu \alpha} c_{\nu \beta} \\
& -d=e^{4 \Phi_{0}} \eta^{\alpha \delta} \eta^{\beta \gamma} c_{\alpha \beta} c_{\delta \gamma}
\end{aligned}
$$

These equations are compatible only if

$$
d=\frac{d(d-2)}{2}
$$

which fixes uniquely

$$
d=4
$$

which seems worth noticing. On the other hand, the other equations of the set (12) are equivalent to

$$
c_{0 i}^{2}=-c_{j k}^{2}
$$

where $i, j, k=1,2,3$ in cyclic order. It is precisely this condition that forbids a minkowskian solution, if the Kalb-Ramond field is to be real.

Let us define the useful combination

$$
c^{2} \equiv \sum_{i} c_{0 i}^{2}
$$

The only remaining equation relates this scale with the value of the dilaton at the origin:

$$
c^{2}=-4 q e^{-4 \Phi_{0}}
$$

The gravitational part of this solution is just $\operatorname{AdS}$ with radius $R=\frac{1}{Q}=\sqrt{\frac{2 \alpha^{\prime}}{7}}$; whereas the dilatonic part is exactly the non-critical linear dilaton; to combine the two in a non-critical background it has been necessary to turn on the antisymmetric field, which in turn is only possible in Euclidean signature. P

\footnotetext{
${ }^{3}$ A similar $A d S$ solution with B-field was found in [4]
} 


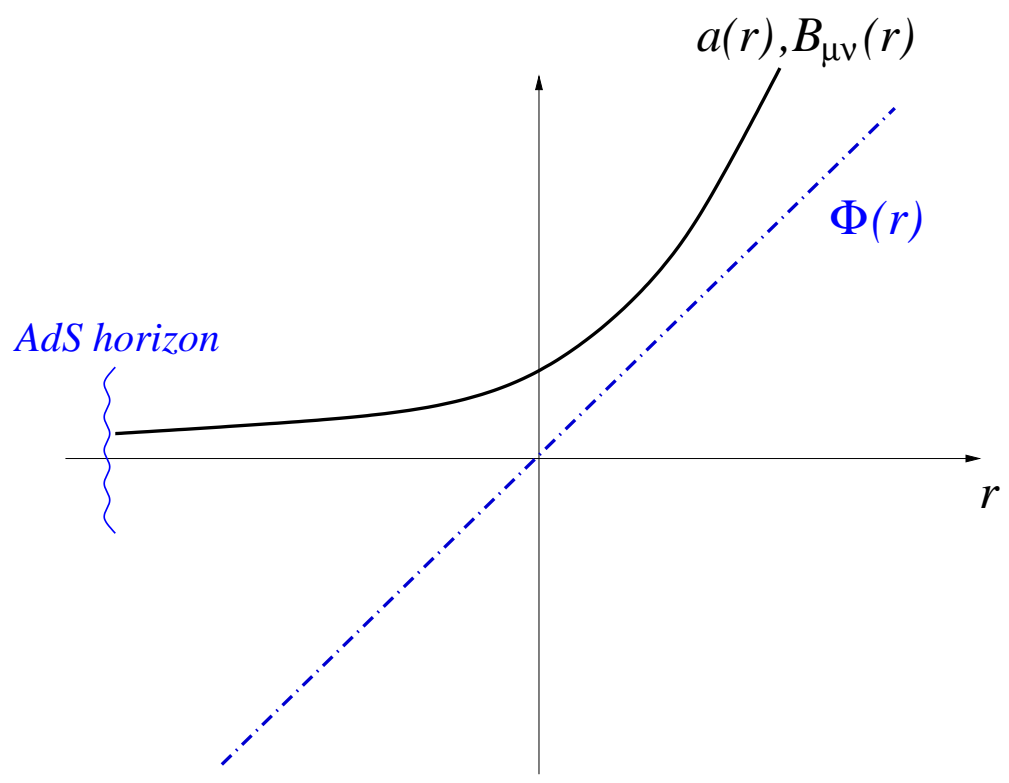

Figure 1: Warp factor, dilaton and Kalb-Ramond field solution v.s. holographic coordinate

There are some simple generalizations of this solution. First of all, we have considered for the time being the bosonic string only, but nothing prevents us to assume world sheet supersymmetry (wss). This changes the value of $q$ only, to $q=\frac{d-9}{4 \alpha^{\prime}}=-\frac{5}{4 \alpha^{\prime}}$, in such a way that the new radius of AdS is $\sqrt{\frac{4 \alpha^{\prime}}{5}}$.

A different generalization involves adding a Ricci flat internal manifold coordinatized by $y$ :

$$
d s^{2}=a(r) d \vec{x}_{d}^{2}+d r^{2}+d \vec{y}_{d^{\prime}}^{2}
$$

The new value of the constant $q$ is now $q=\frac{d+d^{\prime}-25}{6 \alpha^{\prime}}$, or, with wss, $q=\frac{d+d^{\prime}-9}{4 \alpha^{\prime}}$. It is interesting that by adding spectator dimensions the AdS radius becomes larger.

\section{The Interpolating Background}

The solution discussed in the previous paragraph has a strong coupling region, where the dilaton diverges. It would be interesting to look for a solution which somewhat interpolates 
between the two possible signs of the linear dilaton background and, as such, has a string coupling constant everywhere bounded. If we write the general condition for the vanishing of the Weyl anomaly coefficients (7) with the previous ansatz we get the equations relating the conformal factor of the metric with the constant characterizing the Kalb Ramond background:

$$
\begin{aligned}
& -\frac{2 a a^{\prime \prime}+(d-2)\left(a^{\prime}\right)^{2}}{4 a}+a^{\prime} \Phi^{\prime}+\frac{1}{2 a} c^{2} e^{4 \Phi}=0 \\
& -\frac{d}{4} \frac{2 a a^{\prime \prime}-\left(a^{\prime}\right)^{2}}{a^{2}}+2 \Phi^{\prime \prime}+\frac{d c^{2}}{4 a^{2}} e^{4 \Phi}=0 \\
& q+\left(\Phi^{\prime}\right)^{2}-\frac{1}{2} \Phi^{\prime \prime}-\frac{d}{4} \frac{a^{\prime}}{a} \Phi^{\prime}+\frac{d c^{2}}{8 a^{2}} e^{4 \Phi}=0
\end{aligned}
$$

It is a simple matter to check that an euclidean solution is obtained provided $d=4$ and

$$
a(r)=\frac{e^{2 Q r}}{1+2 Q c^{\prime} e^{2 Q r}}
$$

where $c^{\prime}$ is a new constant with dimension of a length. The dilaton is given by:

$$
\Phi=\Phi_{0}+\log a-Q r
$$

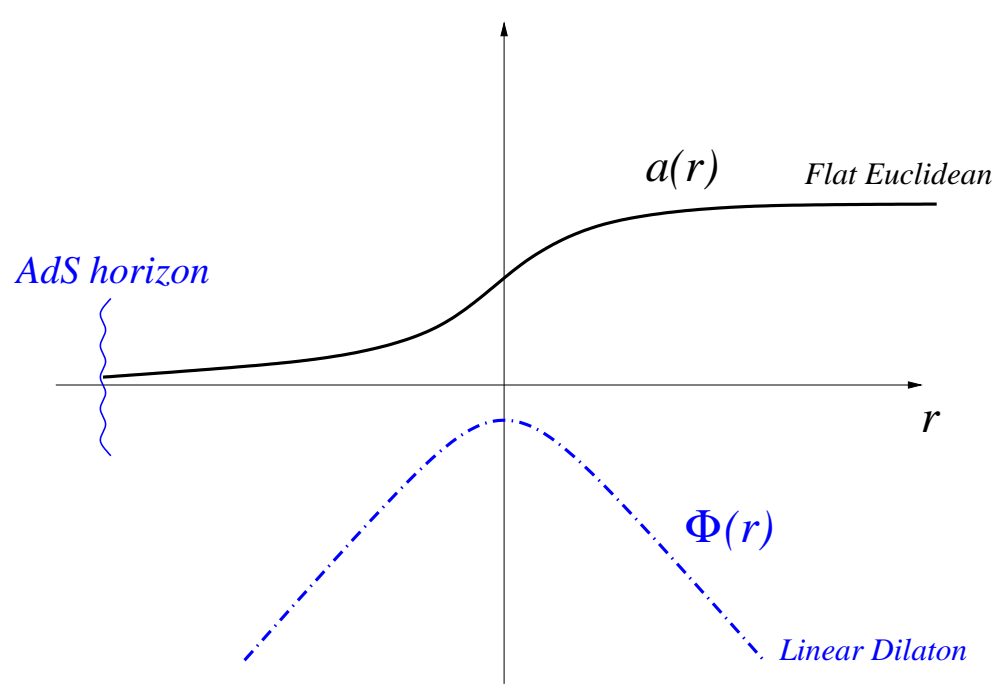

Figure 2: Warp factor and dilaton of the interpolating background 
and the Kalb Ramond field strength is just

$$
H_{4 \mu \nu}=2 Q \frac{c_{\mu \nu}}{c} a^{2} e^{-2 Q r}
$$

with $c$ defined above in Equations (16) and (17).

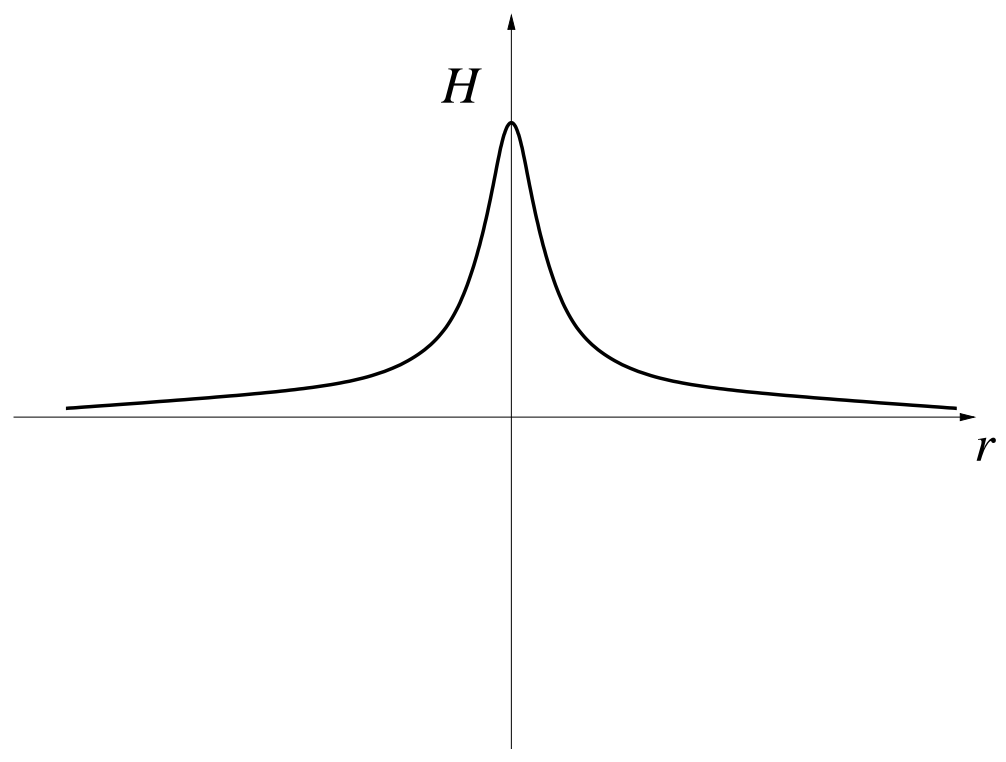

Figure 3: Kalb-Ramond field strengh shape

This new solution reduces to the noncritical AdS background of the previous paragraph when $c^{\prime}=0$. On the other hand, when $c^{\prime} \neq 0$ the dilaton background is of the interpolating type, because

$$
\lim _{r \rightarrow \pm \infty} \Phi(r)=\mp Q r
$$

Several characteristics of the solution are summarized in the Figures 2,3 and 4. Notice, in particular, that each component of the Kalb Ramond field strength has a gaussiam form, with the location of the maximum fixed at $\bar{r} \equiv-\frac{1}{4 Q} \log \left(4 Q^{2}\left(c^{\prime}\right)^{2}\right)$, and height $\bar{H}_{\mu \nu} \equiv \frac{c_{\mu \nu}}{4 c c^{\prime}}$.

There are two features worth discussing. The first is that the solution is singularity free. If the scalar curvature is plotted (cf. Figure 4), it can be easily seen that there is a maximum curvature $R_{\max }=\frac{16 Q^{2}}{9}$ as well as a negative minimum value, given by $R_{\min }=-20 Q^{2}:$ 


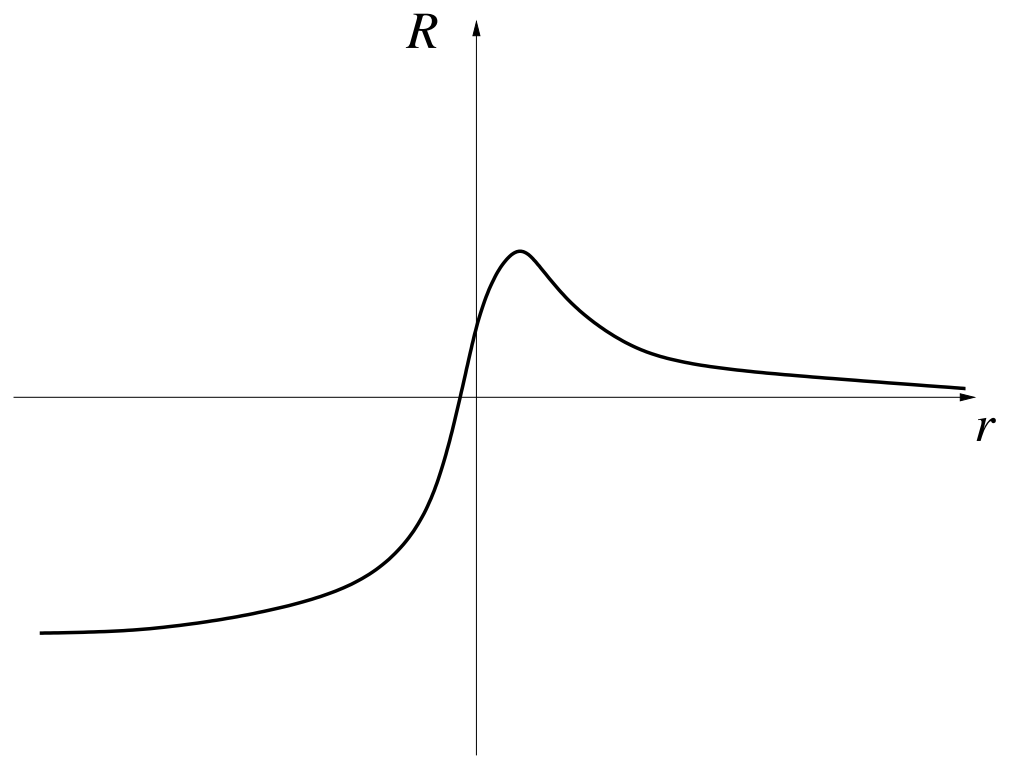

Figure 4: Curvature scalar. Transition between AdS and flat space is observed

This means that there is some hope of not to be led astray by world-sheet sigma model computations. Higher orders in $l_{s}$ could, however, become important as soon as the curvature is big when measured in string units (that is, $R l_{s}^{2} \geq 1$ ).

The second comment refers to the form of the graph of the dilaton in Figure 2. This means that the construct

$$
g_{s} \equiv e^{\Phi}
$$

which is usually referred to as the string coupling is always small

$$
g_{s} \leq 1
$$

In spite of the fact that the string coupling is always small, tadpoles at higher genus can modify our solution.

An interesting characteristic of the solution is the B-field action

$$
I \equiv \frac{1}{12} \int d r d^{4} x \sqrt{g} e^{-2 \Phi} H^{2}
$$


which can be easily computed to be

$$
I=\frac{V_{4}}{c^{\prime}}
$$

where $V_{4}$ is the four dimensional euclidean volume. The corresponding density is then a well defined concept, which vanishes for the linear dilaton and diverges in the pure AdS background.

Actually, vanishing of the Weyl anomaly coefficients leads to

$$
\frac{1}{12} e^{-2 \Phi} H^{2}=-\nabla_{A}\left(e^{-2 \Phi} \nabla^{A} \Phi\right)+2 q e^{-2 \Phi}
$$

This suggests that $I$ can be used as a way to measure the topological change involved in passing from $\Phi=Q r$ at $r=-\infty$ to $\Phi=-Q r$ at $r=+\infty$. The generalization to world sheet supersymmetry and/or flat spectator dimensions is straightforward.

Finally, if a geometric c-function is defined (cf.[2]) the monotonic behavior depicted in Figure 5 is obtained.

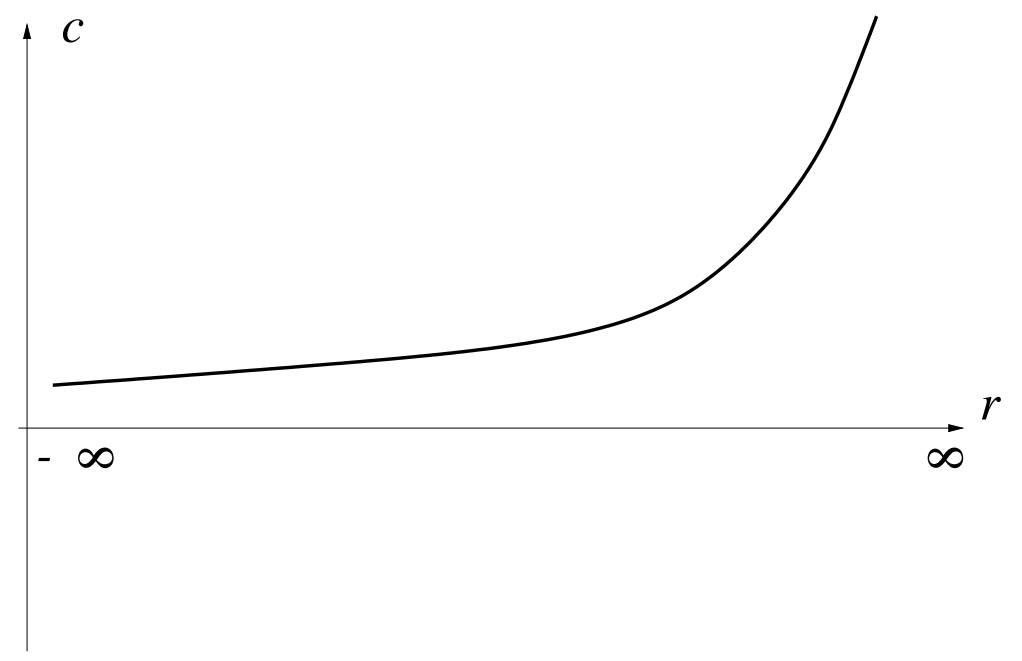

Figure 5: c-function behaviour 


\section{Comments on Closed tachyons}

It has often been argued that a closed tachyon condensate is in some sense equivalent for the string as being non-critical, the precise relationship being given by the Weyl anomaly coefficient

$$
q+(\nabla \Phi)^{2}-\frac{1}{2} \nabla^{2} \Phi-\frac{1}{24} H^{2}+\frac{m^{2}}{16} T^{2}=0
$$

(where $m^{2} \alpha^{\prime}=-4$ for the closed string tachyon).

If we assume world sheet supersymmetry (wss), the only changes are in the numerical value of $\mathrm{q}, q=\frac{D-10}{4 \alpha^{\prime}}$ and the tachyon mass, $m^{2} \alpha^{\prime}=-2$. Then the tachyon potential is guaranteed to be even in $T$.

The preceding equation (29) depends only on the combination

$$
\Delta c \equiv q+\frac{m^{2}}{16} T^{2}=\frac{D-6 T^{2}-26}{6 \alpha^{\prime}}
$$

A vacuum expectation value (vev) for the tachyon is equivalent, from this point of view, to a lower dimension. Actually, our solution trivially generalizes to this case, just by changing $Q \equiv \sqrt{-q}$ by $\sqrt{-\Delta c}$ everywhere. Of course this does not change the number of geometrical dimensions, which remains fixed to $d=4$ (plus the holographic coordinate).

In order to anlyze tachyons in $A d S$ we can use the Breitelohner and Freedman [3] bound

$$
-\frac{1}{4}(D-1)^{2} \leq m^{2} R^{2}
$$

which for the $A d S_{5}$ solution of section 2 gives

$$
m^{2} \geq-\frac{14}{\alpha^{\prime}}
$$

This is not exactly true, due to dilatonic effects. The relevant lagrangian for a scalar field in the dilatonic $A d S$ background of section 2 is

$$
S \sim \int d(v o l) e^{-2 \Phi} \frac{1}{2}\left(\nabla_{A} T \nabla^{A} T-m^{2} T^{2}\right)
$$


In our case the $\sqrt{g}$ factor produces an effect $e^{2 Q r}$, whereas the dilaton term behaves as $e^{-2 Q r}$.

The explicit form of the action is then

$$
S \sim \int d^{4} x d r \frac{1}{2} e^{2 Q r}\left(e^{-2 Q r}\left(\partial_{\mu} T\right)^{2}+\left(\partial_{r} T\right)^{2}-m^{2} T^{2}\right)
$$

For radial fluctuations $\left(\partial_{\mu} T=0\right)$ the net effect is just a change of radius in AdS, from $R=\frac{1}{Q}$, which is the true radius of AdS, to an effective non-dilatonic one, namely $R_{e}=\frac{2}{Q}$. All generic effects of fields propagating in AdS spaces remain the same. Using $R_{e}$ the bound becomes

$$
m^{2} \geq-\frac{21}{6 \alpha^{\prime}}
$$

exactly the bound corresponding to the linear dilaton background. As it is clear from this bound the closed string tachyon of $m^{2}=-\frac{4}{\alpha^{\prime}}$ produces an instability.

Let us now assume, for the sake of the discussion, that the tachyon vev complies with Breitelohner-Freedman bound, that is $T^{2} \geq 1 / 2(6)$ (where the latter figure stands for the wss case). We then would have a five dimensional $A d S$ background where the closed string tachyon becomes of positive energy; i.e a good tachyon (in this case the bound (34) becomes $\left.m^{2} \geq-\frac{4}{\alpha^{\prime}}\left(-\frac{2}{\alpha^{\prime}}\right)\right)$.

In this framework we can now use the interpolating metric to define a tunneling amplitude between both tachyonic vevs. The physical effect of the ensuing tunnelling would be to "restore the symmetry" in the real vacuum:

$$
<0|T| 0>=0
$$

This real vacuum, $\mid 0>$ would be just a quantum mechanical superposition, and, as such, not a solution of the classical equations of motion (in the same sense that the $\theta$-vacuum in QCD is not a classical solution of the Yang-Mills equations). Giving our lack of ability to determine the form of the closed tachyon potential, the above should be taken as a possible scenario only. 


\section{Acknowledgments}

This work has been partially supported by the European Union TMR program FMRXCT96-0012 Integrability, Non-perturbative Effects, and Symmetry in Quantum Field Theory and by the Spanish grant AEN96-1655. The work of E.A. has also been supported by the European Union TMR program ERBFMRX-CT96-0090 Beyond the Standard model and the Spanish grant AEN96-1664.The work of L.H. has been supported by the spanish predoctoral grant AP99-4367460. The work of P.R. has been supported in part by a UAM postgraduate grant.

\section{References}

[1] O. Aharony, M. Berkooz, D. Kutasov and N. Seiberg, Linear dilatons, NS5-branes and holography, JHEP9810 (1998) 004 hep-th/9808149.

[2] E. Alvarez and C. Gomez, Holography and the C-theorem in Contributed to 4th Annual European TMR Conference on Integrailbity, Nonperturbative Effects and Symmetry in Quanum field Theory, Paris, France, 7-13 Sep 2000. hep-th/0009203.

[3] P. Breitelohner and D. Freedman, Stability in gauge extended supergravity, Ann. Phys. $144(1982) 249$.

[4] J. de Boer and S. Shatashvili, Two-dimensional Conformal Field Theories on AdS $S_{2 d+1}$ Backgrounds, JHEP (1999) 9906:013 hep-th/9905032.

[5] L. Girardello, M. Porrati, M. Petrini and A. Zaffaroni, Novel local CFT and exact results on perturbations of $N=4$ super Yang Mills from AdS dynamics, JHEP 9812(1998)022. hep-th/9810126.

[6] S. Gubser, I. Klebanov and A.M. Polyakov, Gauge thepry correlators from noncritical string theory, Phys. Lett.B428 (1998)105. hep-th/9802109. 
[7] J. Maldacena, The Large N limit of superconformal field theories and supergravity, Adv.Theor.Math.Phys 2 (1998) 231-252 hep-th/9711200

[8] R. Myers, New dimensions for old strings, Phys.Lett B 199 (1987) 371

[9] L. Randall and R. Sundrum, A Large mass Hierarchy froma a small extra dimension, Phys.Rev.Lett83 (1999) 3370-3373 hep-th/9905221. ; An alternative to compactification, Phys.Rev.Lett83:4690-4693 (1999) hep-th/9906064.

[10] E. Witten, AdS space and holography, Adv.Theor.Math.Phys2 (1998) 253-291 hepth/9802150.

Anti-de Sitter space, thermal phase transition, and confinement in gauge theories, Adv. Theor. Math. Phys. 2 (1998) 505 hep-th/9803131. 